\title{
Lambert's problem for exponential sinusoids
}

\author{
Dario Izzo*
}

\section{Introduction}

Low-thrust trajectories are becoming increasingly important in the design of space missions. The technological developments that allowed ion propulsion to become a competitive option for missions such as Smart-1 and Deep Space 1 may be soon available for many other advanced propulsion systems that promise to deliver long duration thrust with high specific impulses and high reliability. Methods used to find the optimal trajectories for spacecraft equipped with low-thrust capabilities, are based on direct and indirect approaches highly dependent on the initial guess solution. These approaches, though successful in the detailed design of trajectories, fail in providing the designers with a tool to efficiently explore a large number of options. With this respect the use of analytical methods able to create solutions to the low-thrust problem, such as that suggested by Markopoulos, ${ }^{1}$ could make a difference. In his Ph.D. thesis Petropoulos ${ }^{2}$ introduced for the first time a new analytical solution to the problem of low-thrust trajectories. His solution, based on the use of an exponential sinusoid, is based on an inverse dynamical calculation that leads to analytical expressions both for the thrusting history and for the derivative of the polar anomaly, whenever tangential thrust is assumed. His discovery added up to the small list of analytical solutions available for this increasingly important problem. Moreover, his shape based method, inspired many other researchers. Vasile et al. ${ }^{3}$ tried to solve the problem of constraint violations at the trajectory boundaries (likely to happen with the exponential sinusoid) by introducing shapes defined by a larger number of parameters and by using an equinoctial element formulation. Their method is able to represent with higher fidelity a generic low-thrust trajectory at the cost of an increased number of free parameters. In the same direction is the work by Patel et al. ${ }^{4}$ where an attempt is made to optimise a large class of shapes given in cartesian coordinates. These last approaches constitute a compromise that allow a search through

${ }^{*}$ Research Fellow, Advanced Concepts Team, European Space Agency, ESTEC, Keplerlaan 1, 2201 AZ Noordwijk, The Netherlands. Email: dario.izzo@esa.int 
reasonably large sections of the solution space. When the problem gets complicated, though, these methods suffer from the "curse of dimensionality" and result in a slow search. As recently shown by Myatt et al. ${ }^{5}$ the global trajectory optimisation problem in the case of simpler, ballistic multiple-gravity-assist trajectories may be efficiently treated by the implementation of pruning techniques exploiting the domain knowledge. The same result could be reached also in the low-thrust case, if an equivalent to the Lambert problem solver could be found. The exponential sinusoids found by Petropoulos are a perfect candidate to carry out this task. In a number of works, culminated in the 2004 paper, ${ }^{6}$ Petropoulos and Longuski seek an application of their new trajectories to the interplanetary transfer problem. Their proposed use of exponential sinusoids is tailored to an algorithmic integration into the pre-existing STOUR software. Though quite successful from a numerical point of view, their work did not look at the possible use of advanced global optimisation algorithms in connection with exponential sinusoids. The lack of a discussion on the possibility of using these solutions to go from a generic point $P_{1}$ to another point $P_{2}$ in a given fixed amount of time was a consequence of the use they intended to make of these solutions. It is well known that, in the case of ballistic arcs, this problem, named "Lambert's problem" admits a number of solutions equal to $1+2 \mathrm{~N}$, $N=1,2, \ldots$ as soon as a "short way" or a "long way" gets selected. ${ }^{7}$ In what follows we will investigate what we call the multi-revolution Lambert's problem for exponential sinusoids. When referring to the classical Lambert's problem we will instead explicitly state that we are considering ballistic arcs, i.e. conic sections.

\section{Petropoulos Exponential Sinusoids}

In this section we briefly review the definition of the exponential sinusoids and we comment upon the use of these trajectories made in the STOUR-LTGA program. ${ }^{6}$ Let us start from the equation of motion of a point mass in a Newtonian gravity field under the hypothesis of some added thrust acting on the particle:

$$
\left\{\begin{array}{l}
\ddot{r}-r \dot{\theta}^{2}+\mu / r^{2}=F \sin \alpha \\
\ddot{\theta} r+2 \dot{\theta} \dot{r}=F \cos \alpha
\end{array}\right.
$$

Petropoulos' idea is to see whether an exponential sinusoid, defined by the equation:

$$
r=k_{0} \exp \left[k_{1} \sin \left(k_{2} \theta+\phi\right)\right]
$$


solve the equation of motion Eq.(1). It turns out that under the hypothesis of tangential thrust, i.e. $\alpha=\gamma$, the thrust history and the polar angle history are uniquely determined for each exponential sinusoid by some analytical expressions. These expressions link $\dot{\theta}^{2}$ and $a=F /\left(\mu / r^{2}\right)$ to the four parameters $\left[k_{0}, k_{1}, k_{2}, \phi\right]$ defining each exponential sinusoid. They were derived by Petropoulos ${ }^{2}$ and are reported here for the sake of completeness:

$$
\begin{gathered}
\dot{\theta}^{2}=\left(\frac{\mu}{r^{3}}\right) \frac{1}{\tan ^{2} \gamma+k_{1} k_{2}^{2} s+1} \\
a=\frac{\tan \gamma}{2 \cos \gamma}\left[\frac{1}{\tan ^{2} \gamma+k_{1} k_{2}^{2} s+1}-\frac{k_{2}^{2}\left(1-2 k_{1} s\right)}{\left(\tan ^{2} \gamma+k_{1} k_{2}^{2} s+1\right)^{2}}\right]
\end{gathered}
$$

where the flight path angle is defined by:

$$
\tan \gamma=k_{1} k_{2} \cos \left(k_{2} \theta+\phi\right)
$$

and $s=\sin \left(k_{2} \theta+\phi\right)$. Some analysis reveals that these expression are meaningful whenever $\left|k_{1} k_{2}^{2}\right|<1$ in which case the entire exponential sinusoid shape may be followed by using only a tangential thrust. The original use made of these new trajectories to locate interplanetary transfers, the velocity at departure is considered to be known which, in turn, reduces the definition of the exponential spiral to one free parameter which is chosen to be $k_{2}$. This parameter is then located, with a somewhat complicated procedure, to target the following planet. The resulting algorithm is able to efficiently locate interesting near-optimal solutions, but it makes use of an enumerative search to locate the "good" trajectories. In the 1st Advanced Concepts Team (ACT) Global Trajectory optimisation competition, ${ }^{8}$ exponential sinusoids resulted to be able to locate the basin of attraction of the best solution found to the problem considered.

\section{Another point of view}

In this section we introduce the multi-revolution Lambert's problem for exponential sinusoids. Given $r_{1}, r_{2}$ and the transfer angle $\psi$, we want to find all the exponential sinusoids, defined by Eq.(2), that link the two positions in a given transfer time $t$, allowing for multiple revolutions. We start by reducing the search space assuming $k_{2}$ fixed. We therefore study the problem for all the exponential sinusoids defined by only the three free parameters $k_{0}, k_{1}$ and $\phi$. Without loss of generality we fix our polar coordinate system so that $\theta_{1}=0$. We then write Eq.(5) in correspondence to the first point:

$$
\tan \gamma_{1}=k_{1} k_{2} \cos \phi
$$


It is convenient to use $\gamma_{1}$ i.e. the flight path angle at $r_{1}$, instead of $\phi$ to parameterize the exponential sinusoids. We are only interested in those trajectories that actually do pass by the points $r_{1}$ and $r_{2}$. We enforce this by writing the relations:

$$
\left\{\begin{array}{l}
r_{1}=k_{0} \exp \left[k_{1} \sin \phi\right] \\
r_{2}=k_{0} \exp \left[k_{1} \sin \left(k_{2} \bar{\theta}+\phi\right)\right]
\end{array}\right.
$$

where $\bar{\theta}=\psi+2 \pi N$ and $N=0,1,2, \ldots$, accounts for the possibility of having more than one revolution. By dividing the two equations and taking the logarithm we may derive first the expression:

$$
\frac{k_{1}}{\left|k_{1}\right|} \sqrt{k_{1}^{2}-\frac{\tan ^{2} \gamma_{1}}{k_{2}^{2}}}=\frac{\ln \frac{r_{1}}{r_{2}}+\frac{\tan \gamma_{1}}{k_{2}} \sin k_{2} \bar{\theta}}{1-\cos k_{2} \bar{\theta}}
$$

that tells us what the sign of $k_{1}$ has to be, and then the following:

$$
k_{1}^{2}=\left(\frac{\ln \frac{r_{1}}{r_{2}}+\frac{\tan \gamma_{1}}{k_{2}} \sin k_{2} \bar{\theta}}{1-\cos k_{2} \bar{\theta}}\right)^{2}+\frac{\tan ^{2} \gamma_{1}}{k_{2}^{2}}
$$

We also have, trivially:

$$
\phi=\arccos \left(\frac{\tan \gamma_{1}}{k_{1} k_{2}}\right)
$$

and,

$$
k_{0}=\frac{r_{1}}{\exp \left(k_{1} \sin \phi\right)}
$$

We note that there is no loss of information in considering the angle $\phi$ in the first two quadrants as the consideration of other possibility would simply represent the very same exponential sinusoid given that the sign of $k_{1}$ is also changed. Given the geometry of the problem, i.e. given $r_{1}, r_{2}$ and $\psi$ and given the number of revolutions required, i.e. given $N$ we are now able to say that $\forall k_{2}$ there exists a class $\mathcal{S}_{k_{2}}\left[r_{1}, r_{2}, \psi, N\right]$ of exponential sinusoids passing through $r_{1}$ and $r_{2}$ and conveniently parameterized by the sole free parameter $\gamma_{1}$. The analogy with the theoretical developments that are faced when solving Lambert's problem for ballistic arcs is worth being noted. Also in that case $^{7}$ we arrive at showing that there exist a whole family of ellipses/hyperbolae connecting two given points and we parameterize them conveniently either via the semi-major axis or, even better, by some other alternative variable. 


\section{Introducing the dynamic feasibility}

All that we did in the previous section is only geometrical and there is no information yet introduced on the dynamics. We now ask ourselves which of the exponential sinusoids belonging to $\mathcal{S}_{k_{2}}\left[r_{1}, r_{2}, \psi, N\right]$ are actually feasible trajectories for spacecraft. We call an exponential sinusoid feasible whenever it is possible to find a tangential thrust history able to induce that entire trajectory. Given in these terms the answer is already contained in Petropoulos' Ph.D. dissertation, ${ }^{2}$ the condition is $\left|k_{1} k_{2}^{2}\right|<1$. We may rewrite this condition exploiting Eq.(7):

$$
\left(\frac{k_{2}^{2} \ln \frac{r_{1}}{r_{2}}+k_{2} \tan \gamma_{1} \sin k_{2} \bar{\theta}}{1-\cos k_{2} \bar{\theta}}\right)^{2}+k_{2}^{2} \tan ^{2} \gamma_{1}<1
$$

Some algebra reveals that this is a quadratic equation in $\tan \gamma_{1}$, an equation that allows us to define analytically the interval of $\gamma_{1}$ for which the exponential sinusoids in $\mathcal{S}_{k_{2}}$ are feasible. We find:

$$
\tan \gamma_{1_{m, M}}=\frac{k_{2}}{2}\left[-\ln \frac{r_{1}}{r_{2}} \cot \frac{k_{2} \bar{\theta}}{2} \pm \sqrt{\Delta}\right]
$$

where:

$$
\Delta=\frac{2\left(1-\cos k_{2} \bar{\theta}\right)}{k_{2}^{4}}-\ln ^{2} \frac{r_{1}}{r_{2}}
$$

Trivially if $\Delta<0$ no exponential sinusoid belonging to $\mathcal{S}_{k_{2}}$ is a feasible trajectory. If $\Delta>0$ feasible exponential sinusoids exist for values of $\tan \gamma_{1}$ lying between the two roots given by Eq.(11).

As an example of feasible exponential sinusoids we have plotted some examples in Figure 1. In particular we have considered the family $\mathcal{S}_{1 / 2}[1,5, \pi / 2,0]$ and the family $\mathcal{S}_{1 / 4}[1,5, \pi / 2,1]$. All the trajectories shown may be followed by a spacecraft with unlimited thrusting capabilities that thrusts tangentially to the trajectory. The thrust history, as well as the anomaly history, are uniquely determined for each chosen trajectory by Eq.(3) and Eq.(4).

\section{Time of flight}

If, on one hand, there is a close relation between the developments presented in the previous section and those introduced in the classical Lambert's problem for ballistic arcs, on the other hand the differences are also noteworthy. When ellipses are considered to join the starting and ending position, a pure geometrical approach leads to define a minimum energy ellipse that introduces a limit on the semi-major axis of the possible solution. For 

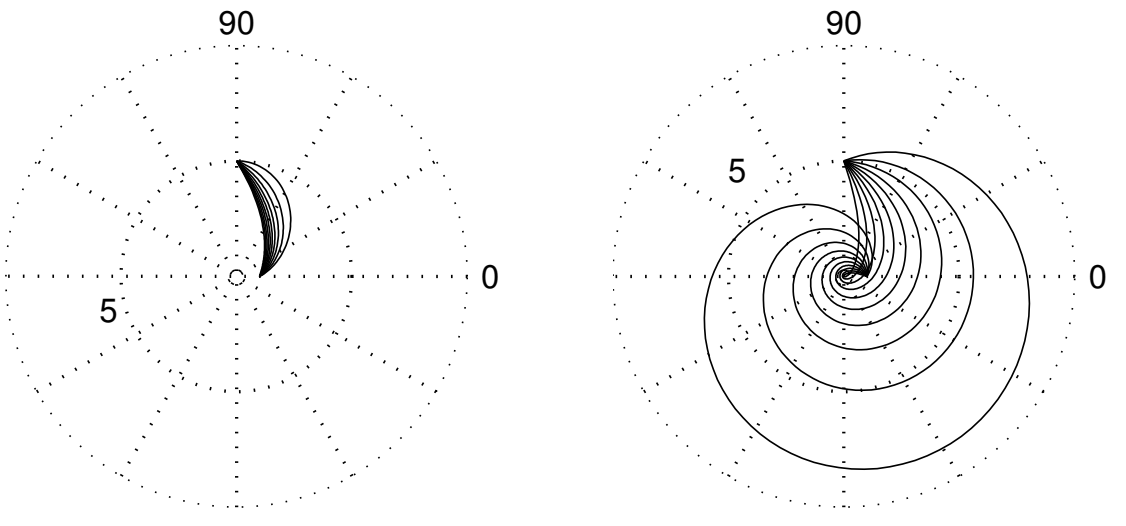

Figure 1. Visualization of all the feasible exponential sinusoids in the classes $\mathcal{S}_{1 / 2}[1,5, \pi / 2,0]$ (left) and $\mathcal{S}_{1 / 4}[1,5, \pi / 2,1]$ (right) 


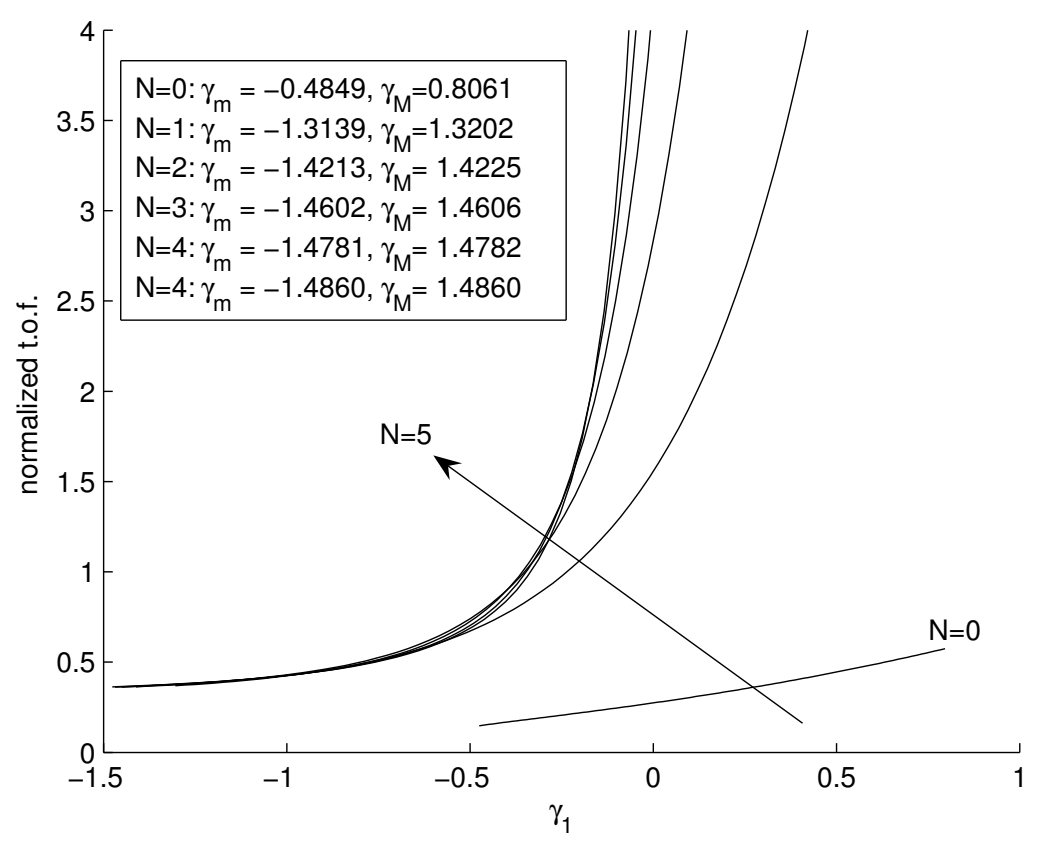

Figure 2. Normalized time-of-flight versus the initial flight-path angle curves for the class $\mathcal{S}_{1 / 12}[1,1.5, \pi / 2, N]$

exponential sinusoids such a limit may be introduced on $\gamma_{1}$, see Eq.(11) only after the dynamics is taken into consideration. In the original problem the time-of-flight equation could be conveniently expressed analytically as a function of the parameter chosen to describe the family of geometrically feasible ellipses. In the case of exponential sinusoids this is not possible and the time-of-fight has to be evaluated by integrating by numerical quadrature the expression given by Eq.(3). Fortunately the chosen parametrization for the exponential sinusoids is a good one and the time of flight curves result to be monotone in $\gamma_{1}$, as shown for a particular case in Figure 2 where a value of $\mu=1$ has been used and the time was normalized with respect to $2 \pi$ (this particular claim has not been demonstrated analytically but it has been thoroughly verified numerically). A very simple numerical method such as Regula Falsi may therefore be used to detect the solution of the Lambert's problem for a particular class of exponential sinusoids by finding the intersection, if existing, between the time-of-flight curve and a horizontal line. We observe that the asymptotic behavior of these time-of-flight curves is quite different from the one we know from the case of ballistic arcs and does not allow to conclude that the problem always has a solution. Also, their being monotone allow us to skip the definition of left branch and right branch necessary in the multi-revolution Lambert's problem for ballistic arcs. 
We may now state, for the exponential sinusoids belonging to a given class $\mathcal{S}_{k_{2}}$, the following equivalent to Lambert's Theorem:

$$
t_{2}-t_{1}=F\left(\tan \gamma_{1}, r_{1}, \frac{r_{1}}{r_{2}}, \bar{\theta}\right)
$$

and we may add that $F$ is a single-valued monotone function of $\gamma_{1}$. In this sense Lambert's problem for exponential sinusoids poses less mathematical challenges than its equivalent for ballistic arcs.

\section{Terminal velocity vectors}

It is well known that in the classic ballistic transfer problem the terminal velocity vectors are closely related. An elegant way of expressing such a relation has been found by Thore Godal (see Battin ${ }^{7}$ Chapter 6) who introduced a skew axis system in which the analytical relationship assumes a noteworthy expression. In the case of exponential sinusoids it is also possible to derive a similar relation. Let us write the expression for the flight-path angle in the final point:

$$
\tan \gamma_{2}=k_{1} k_{2} \cos \left(k_{2} \bar{\theta}+\phi\right)
$$

if we now substitute into this expression Eq.(6) and Eq.(7), after some manipulations it is possible to show that for each sinusoid belonging to a given class $\mathcal{S}_{k_{2}}$ the following relation is valid:

$$
\tan \gamma_{2}=-\tan \gamma_{1}-k_{2} \ln \frac{r_{1}}{r_{2}} \cot \frac{k_{2} \bar{\theta}}{2}
$$

We have established a relation between the terminal velocity vectors, a relation that may be written in an even more compact form if we take into account Eq.(11):

$$
\tan \gamma_{2}=\tan \gamma_{1_{m}}+\tan \gamma_{1_{M}}-\tan \gamma_{1}
$$

This last expression is quite unexpected. It establishes an elegant link between a pure geometrical relation such as that between the terminal flight path angles of all the exponential sinusoids linking two points and two quantities, $\gamma_{1_{m}}$ and $\gamma_{1_{M}}$ whose definition is intrinsically linked to the dynamics of a body in a Newtonian gravitational field. 


\section{A possible application to Orbital Transfers}

Let us consider the design of a multiple gravity assist mission. The goal is to reach a given planet maximizing the final mass of the spacecraft. If only ballistic arcs are allowed to connect the various planets then our decision vector will be $\mathbf{x}=\left\{t_{s}, \Delta t_{i}\right\}$ i.e. the departure date $t_{s}$, the various arc lengths $\Delta t_{i}$. Once these numbers are given, and whenever multiple revolutions are not accounted for, there exist only one possible ballistic trajectory as a consequence of the classical Lambert's theorem. This trajectory may be feasible or unfeasible depending on other mission constraints such as maximum departure velocity (relative to the Earth), minimum pericentrum for the various planet fly-by and others. The problem may be complicated by allowing for multiple revolutions and deep space maneuvers, but in this simple form it has been shown to have a polynomial complexity whenever a particular pruning technique ${ }^{5}$ is used.

When exponential sinusoids are substituted in place of ballistic arcs, we must face the problem of choosing the shape parameter $k_{2}$ for each arc. The simplest case of an interplanetary transfer between two bodies will therefore have a three-dimensional decision vector $\mathbf{x}=\left\{t_{s}, \Delta t_{1}, k_{2}\right\}$. Let us for example take the Earth-Mars case and let us use JPL ephemerides DE405 to evaluate the positions of the planets. Once the decision vector is known we may evaluate the position of the Earth when the spacecraft is launched and the position of Mars at rendezvous. We therefore know $r_{1}, r_{2}, \psi, k_{2}, \Delta t_{1}$ and we may try to locate that unique exponential sinusoid in the class $\mathcal{S}_{k_{2}}\left[r_{1}, r_{2}, \psi, N\right]$ that has $\Delta t_{1}$ as time of flight and therefore to associate a "fitness" to the proposed solution. This opens the possibility of considering a number of global optimisation techniques to find the best possible low-thrust trajectory in the restricted class of the exponential sinusoids.

As a preliminary example we here use the Differential Evolution technique (see Storn and Price $\left.^{9}\right)$ to try locating the global optimum of the following function:

$$
J=\int_{0}^{\bar{\theta}} \frac{|a|}{\dot{\theta}} d \theta
$$

in the space of all the possible exponential sinusoids transferring from Earth to Mars. It is possible to show that this problem is equivalent to the problem of minimizing the ratio $\frac{m_{p}}{m_{s}}$ between the mass of propellant used during the thrust arc and the remaining spacecraft mass $m_{s}$. We select the following bounds for the decision variables (implemented via step penalty functions): $t_{s} \in[2000,5000] \mathrm{MJD}, \Delta t_{1} \in[200,2000]$ days and $k_{2}^{1} \in[.01,1]$. We then search the global optimum in the case of $N=0, N=1$ and $N=2$. Figure 3 depicts 


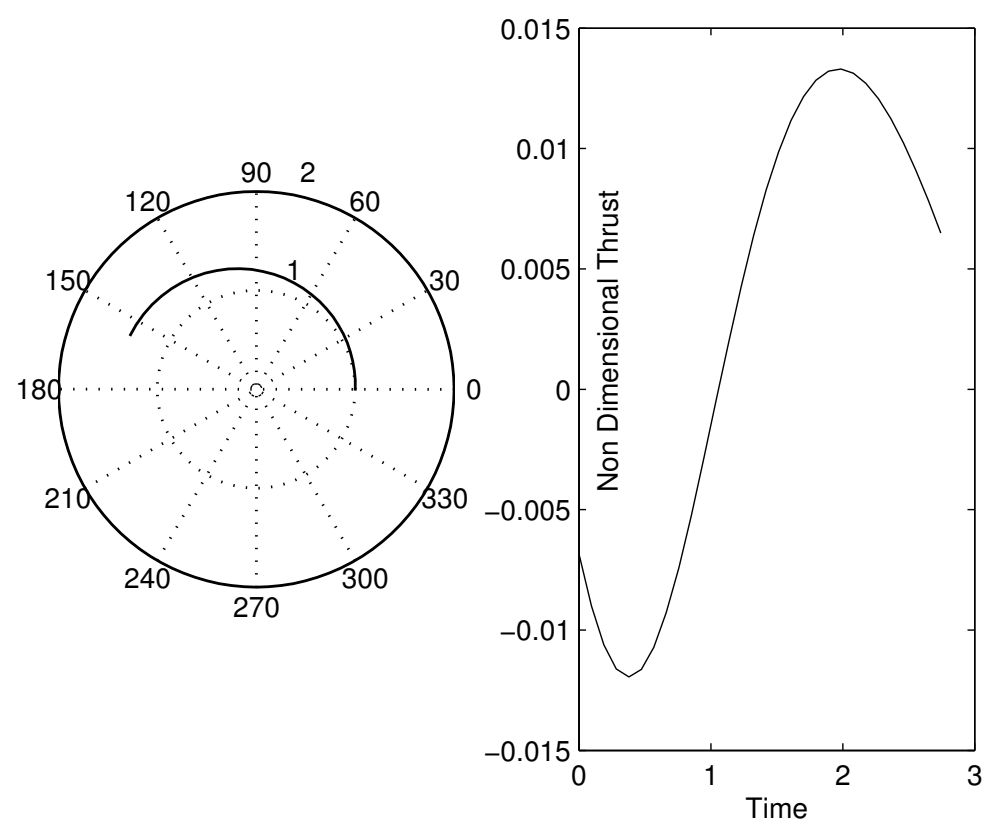

Figure 3. Earth-Mars optimum in the trajectory class of the exponential spirals $N=0$. Units are all non-dimensional

the trajectory found in the case $N=0$ and the non dimensional thrust, while table 1 reports some numerical values for the optimal solutions. The algorithm was run many

\begin{tabular}{cccc}
$\mathrm{N}$ & $t_{s}$ (MJD) & $\Delta t_{1}$ (Days) & $k_{2}^{1}$ \\
\hline \hline 0 & 4115 & 206 & .928 \\
1 & 2034 & 532 & .523 \\
2 & 2021 & 1012 & .2361 \\
\hline
\end{tabular}

Table 1. Global optima for the Earth-Mars case without C3 constraints

times and returned the global optimum roughly $60 \%$ of the times. Other locally optimal solutions were returned in the rest of the trials. The trajectories found are optimal in the class of the exponential sinusoids, it remains to be investigated how rich this class is in terms of its ability of representing possible real solutions. In this particular case, as no constraints were put on the departure and arrival C3, the optimal solution in the wider class of all the possible solutions to Eq.(1) is a ballistic arc, e.g. J=0 and it is not therefore represented by the exponential spirals.

More general cases may also be optimised, i.e. considering gravity assists, in which case the decision vector would be $\mathbf{x}=\left\{t_{s}, \Delta t_{i}, k_{2}^{i}\right\}$ and the optimisation could take into 
account pruning techniques such as those introduced by Myatt et al. ${ }^{5}$ to try to efficiently solve the problem.

\section{Conclusions}

The multi-revolution Lambert's problem has been introduced for the exponential sinusoids and solved. Given the radius ratio the transfer angle and the number of revolutions it has been shown how there exist a family of exponential sinusoids passing through the desired points conveniently parameterized by the flight path angle at the starting point. The functional relationship between such an angle and the time of flight is monotone and its domain is defined by the dynamical feasibility of the resulting trajectories. An example of the use of these results in the search for optimal interplanetary trajectories is given for a simple Earth-Mars transfer.

\section{References}

${ }^{1}$ Markopoulos, N., "Non-Keplerian Manifestation of the Keplerian Trajectory Equation and a Theory of Orbital Motion Under Continuous Thrust," Paper AAS 95-217, Advances in the Astronautical Sciences, Vol. 89, 1995, pp. 1061-1080.

${ }^{2}$ Petropoulos, A. E., Shape-Based Approach to Automated, Low-Thrust,Gravity-Assist Trajectory Design, Ph.D. thesis, School of Aeronautics and Astronautics, Purdue Univ., West Lafayette, IN, 2001.

${ }^{3}$ Vasile, M., Summerer, L., and De Pascale, P., "Design of Earth-Mars transfer trajectories using evolutionary branching techniques," Acta Astronautica, Vol. 56, No. 8, 2005.

${ }^{4}$ Patel, P., Scheeres, D., and Zurbuchen, T., "A Shape Based Approach to Spacecraft Trajectories: Analysis and Optimization," Paper AAS 05-130, Advances in the Astronautical Sciences, Vol. 120, 2005, pp. $445-464$.

${ }^{5}$ Myatt, D., Becerra, V., Nasuto, S., Bishop, J., and Izzo, D., "An Efficient Pruning Technique for the Global Optimisation of Multiple Gravity Assist Trajectories," Proceedings of the International Workshop on Global Optimisation, Almeria, Spain, 18-22 September 2005, pp. 39-46.

${ }^{6}$ Petropoulos, A. and Longuski, J., "Shape-Based Algorithm for Automated Design of Low-Thrust, Gravity-Assist Trajectories," Journal of Spacecrafts and Rockets, Vol. 41, No. 5, 2004.

${ }^{7}$ Battin, R., An Introduction to the Mathematics and Methods of Astrodynamics, Revised Edition, AIAA Education, American Institute of Aeronautics and Astronautics, Inc., 1999.

${ }^{8}$ Izzo, D., "Problem description for the 1st ACT Global Trajectory Optimisation Competition," Tech. rep., ESA, [online] URL: http://www.esa.int/gsp/ACT/doc/ACT-4100-The Problem_V4.pdf [cited 6 February 2006].

${ }^{9}$ Storn, R. and Price, K., "Differential Evolution - a Simple and Efficient Heuristic for Global Optimization over Continuous Spaces," Journal of Global Optimisation, Vol. 11, 1997, pp. 341-359. 